\title{
Deep Convolutional Learning-Aided Detector for Generalized Frequency Division Multiplexing with Index Modulation
}

\author{
Merve Turhan ${ }^{1,2}$, Ersin Öztürk ${ }^{2}$, Hakan Ali Çırpan ${ }^{1}$ \\ ${ }^{1}$ Istanbul Technical University, Faculty of Electrical and Electronics Engineering \\ 34469, Maslak, Istanbul, Turkey \\ Email: \{turhanm17, cirpanh\}@itu.edu.tr \\ ${ }^{2}$ Netas, Department of Research and Development \\ 34912, Pendik, Istanbul, Turkey \\ Email: \{mervet, eozturk\}@netas.com.tr
}

\begin{abstract}
In this paper, a deep convolutional neural network-based symbol detection and demodulation is proposed for generalized frequency division multiplexing with index modulation (GFDM-IM) scheme in order to improve the error performance of the system. The proposed method first pre-processes the received signal by using a zeroforcing (ZF) detector and then uses a neural network consisting of a convolutional neural network $(\mathrm{CNN})$ followed by a fully-connected neural network (FCNN). The FCNN part uses only two fully-connected layers, which can be adapted to yield a trade-off between complexity and bit error rate (BER) performance. This two-stage approach prevents the getting stuck of neural network in a saddle point and enables IM blocks processing independently. It has been demonstrated that the proposed deep convolutional neural network-based detection and demodulation scheme provides better BER performance compared to $\mathrm{ZF}$ detector with a reasonable complexity increase. We conclude that non-orthogonal waveforms combined with IM schemes with the help of deep learning is a promising physical layer (PHY) scheme for future wireless networks.
\end{abstract}

\section{INTRODUCTION}

The demand for reliable, fast and effective wireless communication methods go on with the growing trend thanks to new applications which have challenging technical requirements. In this sense, orthogonal frequency division multiplexing (OFDM) with multiple numerologies concept has been proposed to meet the requested key performance indicators of fifth generation (5G) wireless networks by Third Generation Partnership Project (3GPP) [1], [2]. Although OFDM has solid advantages, e.g., simple equalization, robustness to frequency selective fading and easy implementation, the inabilities of OFDM such as high out-of-band (OOB) emission and high peak-to-average power ratio (PAPR), make it quite disputable to meet the expectations from the physical layer (PHY) of future wireless access technologies [3]. Therefore, improved PHY techniques need to be developed for beyond $5 \mathrm{G}$ wireless networks [4].

Generalized frequency division multiplexing (GFDM) [5] is one of the prominent attempts to cope with the challenges of the future wireless networks. GFDM provides advantages in terms of latency, spectral efficiency, and OOB emission because of block-based structure, reduced overhead of cyclic prefix (CP) and subcarrierbased digitally pulse shaping, respectively. The featured benefit of GFDM is the flexibility that enables timefrequency engineering according to requirements of the target application.

Index modulation (IM) techniques [6] offer energy and spectral efficiency by utilizing transmission entities to convey digital information innovatively. While spatial modulation (SM) [7], [8] utilizes the transmit antennas of a multiple-input multiple-output (MIMO) transmission scheme, OFDM with IM (OFDM-IM) [9]-[11] utilizes the subcarrier indices in a multicarrier system to provide alternative ways for transmitting information. Taking account the efficiencies provided by IM, tight integration of GFDM with IM (GFDM-IM) has been considered and innovative transceiver schemes have been introduced [12]-[17]. In [12], the application of the SM-GFDM system has been considered. In [13], the combination of the IM technique with GFDM has been investigated. In [14], the combination of GFDM with SM and IM techniques has been considered. In [15], a GFDM-based flexible IM transceiver, which is capable of generating and decoding various IM schemes has been proposed. In [16], flexible IM numerology has been proposed to optimize OOB emission, spectral efficiency, and latency jointly. Furthermore, in [17], a novel MIMO-GFDM scheme, which combines spatial multiplexing (SMX) MIMO transmission, GFDM and IM, has been proposed. Despite having optimized transceiver schemes in terms of OOB emission, spectral and energy efficiency, GFDM-IM schemes suffer high computational complexity with respect to conventional OFDM schemes.

Deep learning has recently attracted significant attention because of its high performance to solve computationally-burdened problems in various fields 
such as object detection, natural language processing and computer vision [18]. Considering the unprecedented success of deep learning in classification problems, researchers are eagerly attempting to exploit it for wireless communication. In [19], a pair of blind detectors systems based on the clustering concept has been proposed for SM. In [20], a deep learning-based framework has been presented for channel estimation problem in OFDM. In [21], a zero-forcing (ZF) detector followed by a deep neural network has been proposed for OFDM detection. In [22], a deep complex convolutional network has been developed as an OFDM receiver. In [23] and [24], a communication system has been considered as an autoencoder and communicating binary information through an impaired channel has been treated as reconstruction optimization over impairment layers in a channel autoencoder. This approach has been extended to multi-antenna case in [25]. In [26]-[28], deep learningbased MIMO detection schemes have been proposed. Besides, the use of deep learning has also been considered for uplink/downlink channel calibration in massive MIMO systems [29]. Furthermore, in [30] and [31], deep learning has been exploited for OFDM-IM and GFDM, respectively. For a comprehensive overview of deep learning aided wireless communication, interested readers are referred to [32]-[35].

In this paper, a novel deep convolutional neural network-based detector is proposed for GFDM-IM scheme in order to reduce the complexity while improving error performance. The proposed detector first applies ZF detector to received signal and then uses a neural network, which is composed of a convolutional neural network (CNN) and a fully-connected neural network (FCNN), to recover the transmitted information from the noisy channel outputs. CNN has three important advantages that can help improve a deep learning model in terms of sparse interactions, parameter sharing, and equivariant representations [18]. The FCNN part has only two fully-connected layers, which can be adapted to yield a trade-off between complexity and bit error ratio (BER) performance. To the best of authors' knowledge, the proposed method would be the first attempt to exploit a neural network for GFDM-IM detection. Furthermore, a CNN approach is used to detect IM scheme for the first time. We analyze the uncoded BER performance and computational complexity of the proposed detector by comparing with ZF and maximum likelihood (ML) detectors under Rayleigh multipath fading channels. It has been demonstrated that the proposed scheme provides significant BER improvement compared to ZF detector with a reasonable complexity increase. We conclude that non-orthogonal waveforms combined with IM schemes benefiting deep learning is a promising PHY scheme for future wireless networks.

The remaining sections are organized as follows.
Section II describes the system model. In Section III, deep convolutional neural network-based joint detection and demodulation (JDD) scheme is presented. Section IV analyzes the computational complexity of the proposed detector. Section V evaluates the BER performance of the proposed scheme with respect to the classical linear detectors. Finally, Section VI concludes the paper.

\section{SySTEM MODEL}

Consider a GFDM symbol with $M$ subsymbols each consisting of $K$ subcarriers, the $m$-th subsymbol is partitioned into $L$ IM blocks, each containing $u=K / L$ subcarrier positions. In an IM block, only $v$ out of $u$ subcarrier positions are selected as active and used to transmit quadrature amplitude modulation (QAM) symbols from $Q$-ary signal constellation $\mathcal{S}$ with $Q$ elements. Thus, an IM block can transmit a $p$-bit binary message $\mathbf{s}_{m}^{l}=\left[s_{m}^{l}(1), s_{m}^{l}(2), \ldots, s_{m}^{l}(p)\right]^{T}$. In each IM block, $p_{q}=v \log _{2}(Q)$ bits of incoming $p$-bits sequence are used as QAM-bits. The remaining $p_{i}=\left\lfloor\log _{2}(C(u, v))\right\rfloor$ bits of this sequence are used to determine the active subcarrier positions. Therefore, we have $\alpha=2^{p_{i}}$ possible realizations. Here, $C(\mu, \nu)$ is the binomial coefficient and $\lfloor\cdot\rfloor$ denotes the floor function. Note that active subcarrier positions can be determined using a lookup table or combinatorial methods [9]. As a result, IM blocks $\mathbf{d}_{m}^{l}=\left[d_{m}^{l}(1), d_{m}^{l}(2), \ldots, d_{m}^{l}(u)\right]^{T}$, where $d_{m}^{l}(\gamma) \in\{0, \mathcal{S}\}$, is constructed according to $p$ input bits [13]. Then, IM blocks are first concatenated to obtain the GFDM-IM subsymbol $\mathbf{d}_{m}=\left[d_{0, m}, d_{1, m}, \ldots, d_{K-1, m}\right]$ and the resulting GFDM-IM subsymbols are combined to form the GFDM-IM symbol

$$
\begin{aligned}
& \mathbf{d}= \\
& {\left[d_{0,0}, \ldots, d_{K-1,0}, d_{0,1}, \ldots, d_{K-1,1}, \ldots, d_{K-1, M-1}\right],}
\end{aligned}
$$

where $d_{k, m} \in\{0, \mathcal{S}\}$, for $k=0, \ldots, K-1, m=$ $0, \ldots, M-1$, is the data symbol of $k$-th subcarrier on $m$-th subsymbol. After that, the GFDM-IM symbol $\mathbf{d}$ is modulated using a GFDM modulator and the resulting GFDM transmit signal can be expressed as

$$
\mathbf{x}=\mathbf{A d}
$$

where $\mathbf{A}$ is an $N \times N$ GFDM modulation matrix [5], $N=K M$. Finally, a CP with length $N_{\mathrm{CP}}$ is appended to $\mathbf{x}$ and the resulting vector $\tilde{\mathbf{x}}=$ $\left[\mathbf{x}\left(K M-N_{\mathrm{CP}}+1: K M\right)^{T}, \mathbf{x}^{T}\right]^{T}$ is sent over a frequency-selective Rayleigh fading channel.

At the receiver side, assuming that perfect synchronization is ensured, CP is longer than the tap length of the channel $\left(N_{\mathrm{Ch}}\right)$ and the wireless channel remains constant during the transmission of a GFDM symbol, the received signal vector $\mathbf{y}$ can be expressed as

$$
\mathbf{y}=\mathbf{H x}+\mathbf{n}
$$


after the removal of CP. Here, $\mathbf{y}=$ $[y(0), y(1), \ldots, y(N-1)]^{\mathrm{T}}$ is the vector of the received signals, $\mathbf{H}$ is the $N \times N$ circular convolution matrix constructed from the channel impulse response coefficients given by $\mathbf{h}=\left[h(1), h(2), \ldots, h\left(N_{\mathrm{Ch}}\right)\right]^{\mathrm{T}}$, and $\mathbf{n}$ is an $N \times 1$ vector of additive white Gaussian noise (AWGN) samples. The elements of $\mathbf{h}$ and $\mathbf{n}$ follow $\mathcal{C N}(0,1)$ and $\mathcal{C N}\left(0, \sigma_{w}^{2}\right)$ distributions, respectively, where $\mathcal{C N}\left(\mu, \sigma^{2}\right)$ shows the distribution of a circularly symmetric complex Gaussian random variable with mean $\mu$ and variance $\sigma^{2}$. After substituting Eq. 1 in Eq. 2. we obtain the equivalent channel of the GFDM-IM scheme as

$$
\mathbf{y}=\mathbf{H A d}+\mathbf{n}=\tilde{\mathbf{H}} \mathbf{d}+\mathbf{n}
$$

\section{DeEP Detection And Demodulation}

The block diagram of the proposed deep convolutional neural network-based joint GFDM-IM detection and demodulation scheme, termed as DeepConvIM, is shown in Fig 1. It is assumed that the receiver has the channel information. In contrast to OFDM-IM, GFDMIM subcarriers can be non-orthogonal to each other due to non-rectangular pulse shaping. Therefore, the inherent ICI prevents the frequency domain decoupling of GFDM-IM subcarriers for both single-input singleoutput (SISO) and MIMO transmission schemes. As a result, simultaneous detection of all subcarriers is required for optimum decision. Since this process is computationally infeasible, low complexity solutions are required for the optimum detection problem of GFDMIM. Inspired from [21], the proposed detector has two parts as coarse detector and fine detector. This two stage approach prevents getting stuck of neural network in a saddle point and enables the processing IM blocks independently. First, coarse detector uses ZF detector in order to process channel and GFDM modulation effects jointly. The output vector of coarse detector can be expressed as

$$
\hat{\mathbf{d}}=\left(\widetilde{\mathbf{H}}^{H} \tilde{\mathbf{H}}\right)^{-1} \widetilde{\mathbf{H}}^{H} \mathbf{y}
$$

Since coarse detector operates on the equivalent channel of the GFDM-IM scheme, the remaining parts can handle the IM blocks individually. Therefore, fine detector processes the IM blocks independently. IM Block Splitter partitions the pre-processed received vector $\hat{\mathbf{d}}$ into IM blocks $\hat{\mathbf{d}}_{m}^{l}=\left[\hat{d}_{m}^{l}(1), \hat{d}_{m}^{l}(2), \ldots, \hat{d}_{m}^{l}(u)\right]^{T}$. The fine detector part of DeepConvIM uses a CNN followed by a FCNN, which is expressed as

$$
\hat{\mathbf{s}}_{m}^{l}=\theta\left(\hat{\mathbf{d}}_{\mathbf{m}}^{\mathbf{l}}\right),
$$

where $\theta$ represent the total of trainable parameters. The CNN part of the fine detector convolves the IM block $\hat{\mathbf{d}}_{m}^{l}$ with the kernel filter $\mathbf{a}_{t}=\left[\begin{array}{ll}a_{t, R} & a_{t, I}\end{array}\right]$, adds bias $\mathbf{c}_{t}$, for $t=1, \ldots T$, with stride 1 , and the modified received IM block can be expressed as

$$
\begin{aligned}
& \check{d}_{m, t}^{l}(\gamma)= \\
& \quad \tanh \left(a_{t, R} * \Re\left(\hat{d}_{m}^{l}(\gamma)\right)+a_{t, I} * \Im\left(\hat{d}_{m}^{l}(\gamma)\right)+c_{t}\right),
\end{aligned}
$$

where tanh is an activation function, for $\gamma=1, \ldots, u$. Here $\mathbf{a}_{t}$ and $\mathbf{c}_{t}$ are called convolution trainable parameters. Notice that unlike [30], DeepConvIM does not need the energy of the received signal. The FCNN part of the fine detector gets the output of the CNN and performs deep detection by using $\{\mathbf{W}, \mathbf{b}\}$ trainable parameters, where $\mathbf{W}=\left[\mathbf{w}_{1}, \mathbf{w}_{2}\right]$ contains weights parameters and $\mathbf{b}=\left[b_{1}, b_{2}\right]$ contains bias parameters. That is, The FCNN part uses only two fully-connected layers, hidden layer has $\tau$ nodes the output layer has $p$ nodes as expected. The output of fine detector can be expressed as

$$
\hat{\mathbf{s}}_{m}^{l}=\operatorname{sigmoid}\left(\mathbf{w}_{2}\left(\tanh \left(\mathbf{w}_{1} \check{\mathbf{d}}_{m, t}^{l}+b_{1}\right)\right)+b_{2},\right.
$$

where sigmoid is an activation function. Finally, IM Block Combiner combines the output of the fine detector and forms the transmitted information bits.

The aim of the training stage of DeepConvIM is to find $\theta$ parameters in order to minimize the loss function, which is expressed as $\operatorname{loss}\left(\mathbf{s}_{m}^{l}, \hat{\mathbf{s}}_{m}^{l}\right)=\left\|\mathbf{s}_{m}^{l}-\hat{\mathbf{s}}_{m}^{l}\right\|$. Before training, GFDM-IM simulation training data is generated and divided into batchs $(B)$. At first, the $\theta$ is randomly initialized. Throughout the training, $\theta$ is updated according to stochastic gradient descent (SGD) algorithm for every batch, which is expressed as

$$
\theta_{+}=\theta-\eta \nabla \operatorname{loss}\left(\mathbf{s}_{m}, \hat{\mathbf{s}}_{m}\right)
$$

where $\eta$ is learning rate.

\section{COMPlEXITy ANALYSIS}

Computational complexity of ZF, ML and DeepConvIM detectors is investigated from the standpoint of number of complex multiplications (CMs) and given in Table I] Here, $\Psi_{J \times I}$ and $\Phi_{J \times I}$ are used for $J \times I$ matrices, $\psi_{J \times 1}$ and $\phi_{J \times 1}$ stand for $J \times 1$ vectors. Notice that using complex numbers is not yet supported by any popular deep learning frameworks and FCNN part of DeepConvIM operates on real numbers thanks to $\mathrm{CNN}$ part. Since one complex multiplication can be carried out with at least three real multiplications, the number of multiplications belonging to neural networks parts of DeepConvIM are divided to three in order to refer them as complex multiplications. The summary of the results is given in Table III. From Table II] it is observed that while $\mathrm{ZF}$ and ML detectors have the lowest and the highest complexity, respectively, DeepConvIM provides an intermediate solution with regard to computational complexity. 


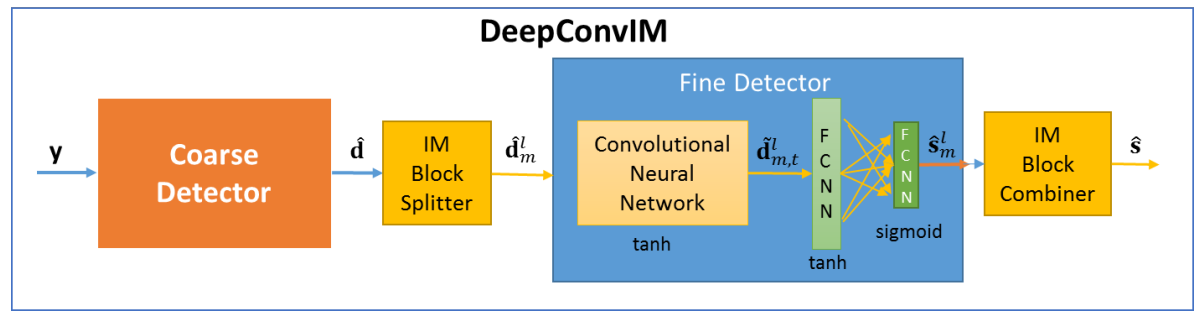

Fig. 1. Block diagram of the deep convolutional neural network-based GFDM-IM detector.

TABLE I

Computational Complexity of ZF, ML And DeepConvim Detectors

\begin{tabular}{|c|c|c|c|c|}
\hline Detector & Process & Operation & Execution Count & Complexity $(\mathrm{CM})$ \\
\hline \multirow{3}{*}{ ZF } & Forming $\tilde{\mathbf{H}}$ & $\Phi_{N \times N} \Psi_{N \times N}^{\dagger}$ & 1 & $N_{\mathrm{Ch}} N^{2}$ \\
\hline & JDD & $\left(\Phi_{N \times N}{ }^{\mathrm{H}} \Phi_{N \times N}\right)^{-1} \Phi_{N \times N}{ }^{\mathrm{H}} \phi_{N \times 1}$ & 1 & $3 N^{3}+N^{2}$ \\
\hline & Decision & $\left.\min \left(\| \phi_{u \times 1}-\psi_{u \times 1}\right) \|^{2}\right)$ & $M L$ & $u \alpha Q^{v} M L$ \\
\hline \multirow{2}{*}{ ML } & Forming $\widetilde{\mathbf{H}}$ & $\Phi_{N \times N} \Psi_{N \times N}^{\dagger}$ & 1 & $N_{\mathrm{Ch}} N^{2}$ \\
\hline & Decision & $\min \left(\left\|\phi_{N \times 1}-\left(\Phi_{N \times N} \psi_{N \times 1}\right)\right\|^{2}\right)^{\dagger \dagger}$ & $\left(\alpha Q^{v}\right)^{M L}$ & $\left(\alpha Q^{v}\right)^{M L}(N v M L+N)$ \\
\hline \multirow{4}{*}{ DeepConvIM } & Forming $\widetilde{\mathbf{H}}$ & $\Phi_{N \times N} \Psi_{N \times N}^{\dagger}$ & 1 & $N_{\mathrm{Ch}} N^{2}$ \\
\hline & JDD & $\left(\Phi_{N \times N}{ }^{\mathrm{H}} \Phi_{N \times N}\right)^{-1} \Phi_{N \times N}{ }^{\mathrm{H}} \phi_{N \times 1}$ & 1 & $3 N^{3}+N^{2}$ \\
\hline & $\mathrm{CNN}$ & $(2 u T+u T \lambda) / 3^{\dagger \dagger \dagger}$ & $M L$ & $(u T(2+\lambda)) M L / 3$ \\
\hline & FCNN & $(u T \tau+\tau \lambda+\tau p+p \delta) / 3^{\dagger \dagger \dagger}$ & $M L$ & $(u T \tau+\tau \lambda+\tau p+p \delta) M L / 3$ \\
\hline
\end{tabular}

${ }^{\dagger}$ In every row of $\mathbf{H}$, which is $\Phi$ in this case, only $N_{C h}$ out of $N$ elements are non-zero.

$\dagger^{\dagger \dagger}$ In $\psi$, only $v M L$ complex elements are nonzero.

${ }^{\dagger \dagger} \lambda \lambda$ refers to number of real multiplications required for tanh function.

${ }^{\dagger \dagger \dagger} \delta$ and $\tau$ refers to number of real multiplications required for sigmoid function and the number of nodes of the hidden layer of FCNN, respectively.

TABLE II

Summary of the Computational Complexity of ZF, ML and DeepConviM Detectors

\begin{tabular}{|l||c|}
\hline Detector & Total Complexity $(C M s)$ \\
\hline \hline ZF & $3 N^{3}+N^{2}\left(1+N_{\mathrm{Ch}}\right)+\alpha Q^{v} M L$ \\
\hline ML & $\left(\alpha Q^{v}\right)^{M L}(N v M L+N)+N_{\mathrm{Ch}} N^{2}$ \\
\hline DeepConvIM & $3 N^{3}+N^{2}\left(1+N_{\mathrm{Ch}}\right)+(u T(2+\lambda)) M L / 3+(u T \tau+\tau \lambda+\tau p+p \delta) M L / 3$ \\
\hline
\end{tabular}

TABLE III

A LOOK-UP TABLE EXAMPLE FOR $u=4, v=2$.

\begin{tabular}{|c|c|c|}
\hline Bits & Indices & IM block \\
\hline$\left[\begin{array}{ll}0 & 0\end{array}\right]$ & $\{1,2\}$ & {$\left[\begin{array}{llll}s_{\chi} & s_{\zeta} & 0 & 0\end{array}\right]^{1}$} \\
\hline$\left[\begin{array}{ll}0 & 1\end{array}\right]$ & $\{2,3\}$ & {$\left[\begin{array}{llll}0 & s_{\chi} & s_{\zeta} & 0\end{array}\right]^{7}$} \\
\hline$\left[\begin{array}{ll}1 & 0\end{array}\right]$ & $\{3,4\}$ & {$\left[\begin{array}{llll}0 & 0 & s_{\chi} & s_{\zeta}\end{array}\right]^{7}$} \\
\hline$\left[\begin{array}{ll}1 & 1\end{array}\right]$ & $\{1,4\}$ & {$\left[\begin{array}{ll}s_{\chi} & 0\end{array}\right.$} \\
\hline
\end{tabular}

\section{NumericAl Results}

In this section, the BER performance of DeepConvIM has been compared to ZF and ML detection methods by computer simulations for Rayleigh fading with Extended Pedestrian A (EPA) channel model [36]. The chosen pulse shape for the GFDM prototype filter is the raised cosine (RC) filter with a roll-off factor $(a)$ of 0.5 . The active subcarrier indices are selected using the lookup table in Table IIII Fine detector model parameters and summary are given in Table $[\mathrm{IV}$ and $\mathrm{V}$, respectively. During training stage, signal-to-noise ratio (SNR) is set to $15 \mathrm{~dB}$, Adam optimizer [37], which is SGD-based, is used, and the learning rate is set to $8 \times 10^{-4}$. The
DeepConvIM model is trained in a short time, 60 epochs is enough to get significant results. A GFDM-IM training data set, including $16 \times 10^{4}$ symbols, and a GFDM-IM testing data set, including $3 \times 10^{4}$ symbols are generated for each SNR value regarding $K=32$. For $K=8$, training data and testing data include $32 \times 10^{4}$ and $6 \times 10^{4}$ GFDM-IM symbols, recpectively.

DeepConvIM model is constructed using Keras [38] (backend Tensorflow [39]) and trained on Google Colab, providing tensor processing units (TPUs) in the cloud environment.

Fig. 2 compares the BER performance of the ZF and the DeepConvIM with ZF coarse detector for binary phase shift keying (BPSK) transmission when $K=32$ and $M=1$. From Fig. 2, it is observed that the DeepConvIM provides approximately $6 \mathrm{~dB}$ better BER performance than $\mathrm{ZF}$ at a BER value of $10^{-4}$.

Fig. 3 compares the BER performance of the ZF and the DeepConvIM with ZF coarse detector for 4QAM and 16-QAM transmissions when $K=32$ and $M=1$. From Fig. 3 it is observed that the DeepConvIM provides approximately 4.5 and $1 \mathrm{~dB}$ better BER perfor- 
TABLE IV

Fine Detector Model Parameters

\begin{tabular}{|l||c|c|}
\hline Description & Parameter & Value \\
\hline \hline Number of Kernel Filter (for BPSK transmission) & $T$ & 16 \\
\hline Number of Kernel Filter (for 4-QAM transmission) & $T$ & 32 \\
\hline Number of Kernel Filter (for 16-QAM transmission) & $T$ & 64 \\
\hline Number of Nodes of Hidden Layer (for BPSK transmission) & $\tau$ & 64 \\
\hline Number of Nodes of Hidden Layer (for 4-QAM transmission) & $\tau$ & 128 \\
\hline Number of Nodes of Hidden Layer (for 16-QAM transmission) & $\tau$ & 256 \\
\hline Learning Rate & $l r$ & 0.0008 \\
\hline Batch Size & $B$ & 1000 \\
\hline
\end{tabular}

TABLE V

Fine Detector Model Summary

\begin{tabular}{|c|c|c|}
\hline Layer & Output Shape & Activation Func. \\
\hline \hline Input & $(\mathrm{B}, 2, \mathrm{u}, 1)$ & None \\
\hline Conv2d & $(\mathrm{B}, 1, \mathrm{u}, \mathrm{T})$ & tanh \\
\hline Flatten & $(\mathrm{B}, \mathrm{uT})$ & None \\
\hline Dense & $(\mathrm{B}, \tau)$ & tanh \\
\hline Dense & $(\mathrm{B}, \mathrm{p})$ & sigmoid \\
\hline
\end{tabular}

mance than ZF for 4-QAM and 16-QAM transmissions, respectively, at a BER value of $10^{-4}$.

Fig. 4 compares the BER performance of the ZF, ML and the DeepConvIM with ZF coarse detector for BPSK transmission, when $K=8, M=(1,3)$. From Fig. 4 . at a BER value of $10^{-4}$, while DeepConvIM provides $6 \mathrm{~dB}$ BER improvement with respect to $\mathrm{ZF}$ detector for $M=3$, the BER improvement of DeepConvIM with ZF coarse detector with respect to $\mathrm{ZF}$ detector is increased to $8 \mathrm{~dB}$ when $M=1$. On the other hand, ML detector has $8 \mathrm{~dB}$ BER improvement with respect to DeepConvIM when $M=1$.

From Fig. 2 and 3 , it is observed that as the modulation order increases, the learning capacity of the model decreases. From Fig. 3, it is observed that when the number of subsymbols increases, performance of the model decreases. On the other hand, from Fig. 2 and 4, it is observed that when the number of subcarriers decreases, performance of the model increases. The number of CMs needed by the detectors in Fig. 2, 3 and 4 are given in Table VI As mentioned earlier, DeepConvIM can be evaluated as a intermediate solution regarding computational complexity.

\section{CONCLUSION}

In this paper, a new GFDM-IM receiver scheme, which is constructed by the combination of a $\mathrm{ZF}$ detector and a deep convolutional neural network, has been proposed. BER performance of the proposed scheme has been compared to $\mathrm{ZF}$ and ML detectors by computer simulations under Rayleigh multipath fading channels. The proposed scheme has very simple and flexible neural network structure, which can be adapted to yield a tradeoff between complexity and BER performance. It has been demonstrated that the proposed scheme provides significant BER improvement compared to $\mathrm{ZF}$ detector with a reasonable complexity increase. We conclude that

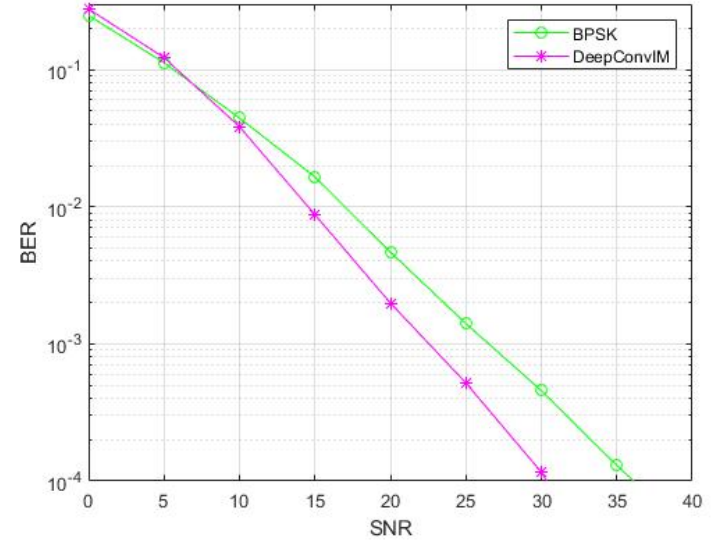

Fig. 2. BER performance of $\mathrm{ZF}$ and DeepConvIM with $\mathrm{ZF}$ coarse detector for BPSK transmission, $(K=32, M=1)$

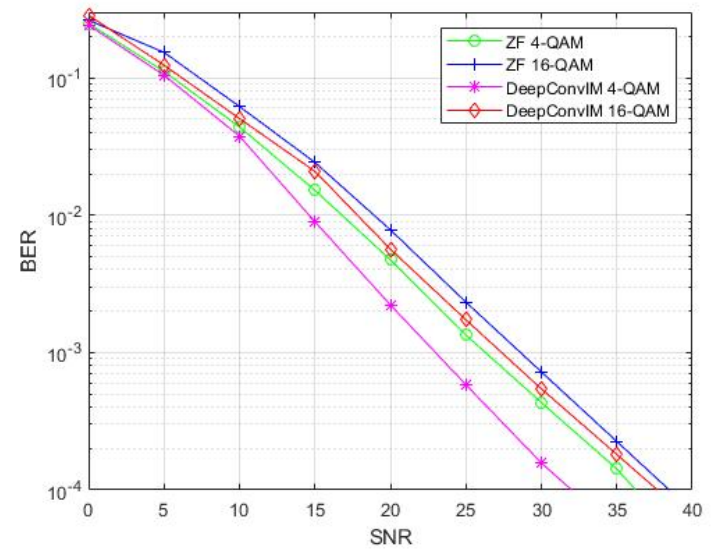

Fig. 3. BER performance of $\mathrm{ZF}$ and DeepConvIM with $\mathrm{ZF}$ coarse detector for 4-QAM and 16-QAM transmissions $(K=32, M=1)$.

deep convolutional learning-aided GFDM-IM scheme can be considered a promising $\mathrm{PHY}$ layer technique for beyond $5 \mathrm{G}$ wireless networks. As a future work, we will study application of deep learning to MIMO-GFDM systems.

\section{REFERENCES}

[1] 3GPP, "NR, physical layer, general description," tech. spec. 38.201, Dec. 2017.

[2] - "Study on new radio (NR) access technologies," tech. rep. 38.912 V14.1.0, Jun. 2017.

[3] G. Wunder et. al., "5GNOW: Non-orthogonal, asynchronous waveforms for future mobile applications," IEEE Commun. Mag., vol. 52, no. 2, pp. 97-105, Feb. 2014. 
TABLE VI

The Total Number of CMs For ZF, ML and DeepConviM Detectors

\begin{tabular}{|l||c|c|c|}
\hline Configuration & $Z F$ & DeepConvIM & $M L$ \\
\hline \hline BPSK, $K=32, M=1$ & $1.07 \times 10^{5}$ & $1.18 \times 10^{5}$ & $2.33 \times 10^{\Gamma 2}$ \\
\hline 4-QAM, $K=32, M=1$ & $1.09 \times 10^{5}$ & $1.56 \times 10^{5}$ & $1.48 \times 10^{22}$ \\
\hline 16-QAM, $K=32, M=1$ & $2.37 \times 10^{5}$ & $4.20 \times 10^{5}$ & $4.15 \times 10^{36}$ \\
\hline BPSK, $K=8, M=1$ & $2.08 \times 10^{3}$ & $5.15 \times 10^{3}$ & $1.07 \times 10^{4}$ \\
\hline BPSK, $K=8, M=3$ & $4.61 \times 10^{4}$ & $5.54 \times 10^{4}$ & $5.23 \times 10^{9}$ \\
\hline
\end{tabular}

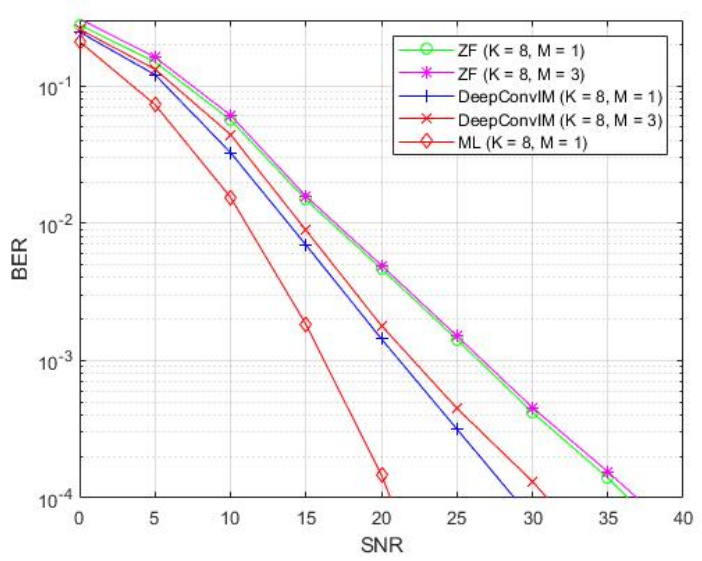

Fig. 4. BER performance of $\mathrm{ZF}$ and DeepConvIM with $\mathrm{ZF}$ coarse detector for BPSK transmission $(K=8, M=(1,3))$.

[4] Z. E. Ankarali, B. Pekoz, and H. Arslan, "Flexible radio access beyond 5G: A future projection on waveform, numerology and frame design principles," IEEE Access, vol. PP, no. pp, pp. 1-1, Mar. 2017

[5] N. Michailow, M. Matthe, I. Gaspar, A. Caldevilla, L. Mendes, A. Festag, and G. Fettweis, "Generalized frequency division multiplexing for 5th generation cellular networks," IEEE Trans. Commun., vol. 62, no. 9, pp. 3045-3061, Sep. 2014.

[6] E. Basar et. al., "Index modulation techniques for next-generation wireless networks," IEEE Access, vol. 5, no. 1, pp. 16693 16746, Sep. 2017.

[7] R. Mesleh, H. Haas, S. Sinanovic, C. W. Ahn, and S. Yun, "Spatial modulation," IEEE Trans. Veh. Technol., vol. 57, no. 4, pp. 2228-2241, Jul. 2008.

[8] M. Di Renzo et. al., "Spatial modulation for generalized MIMO: Challenges, opportunities, and implementation," Proceedings of the IEEE, vol. 102, no. 1, pp. 56-103, 2014.

[9] E. Basar, Ü. Aygölü, E. Panayırc1, and H. V. Poor, "Orthogonal frequency division multiplexing with index modulation," IEEE Trans. Signal Process., vol. 61, no. 22, pp. 5536-5549, Nov. 2013.

[10] R. Abu-alhiga and H. Haas, "Subcarrier-index modulation OFDM," in IEEE Int. Sym. Personal, Indoor and Mobile Radio Commun., Tokyo, Japan, Sep. 2009, pp. 177-181.

[11] D. Tsonev, S. Sinanovic, and H. Haas, "Enhanced subcarrier index modulation (SIM) OFDM," in IEEE GLOBECOM Workshops, Dec. 2011, pp. 728-732.

[12] E. Ozturk, E. Basar, and H. Cirpan, "Spatial modulation GFDM: A low complexity MIMO-GFDM system for 5G wireless networks," in Proc. 4th IEEE Int. Black Sea Conf. Commun. Networking, Varna, Bulgaria, Jun. 2016.

[13] — , "Generalized frequency division multiplexing with index modulation," in Proc. IEEE GLOBECOM Workshops, Washington DC, USA, Dec. 2016.

[14] — , "Generalized frequency division multiplexing with space and frequency index modulations," in Proc. 5th IEEE Int. Black Sea Conf. Commun. Networking, Istanbul, Turkey, Jun. 2017.

[15] — "Generalized frequency division multiplexing with flexible index modulation," IEEE Access, vol. 5, pp. 24727 - 24746, Oct. 2017.

[16] — , "Generalized frequency division multiplexing with flexi- ble index modulation numerology," IEEE Signal Process. Lett., vol. 25 , no. 10 , pp. 1480-1484, Oct. 2018.

[17] — "Multiple-input multiple-output generalized frequency division multiplexing with index modulation numerology," Physical Communication, vol. 34, pp. 27-37, 2019.

[18] I. Goodfellow, Y. Bengio, and A. Courville, Deep Learning. MIT Press, 2016, http://www.deeplearningbook.org

[19] L. You, P. Yang, Y. Xiao, S. Rong, D. Ke, and S. Li, "Blind detection for spatial modulation systems based on clustering," IEEE Communications Letters, vol. 21, no. 11, pp. 2392-2395, Nov 2017.

[20] M. Soltani, A. Mirzaei, V. Pourahmadi, and H. Sheikhzadeh, "Deep learning-based channel estimation," 2018.

[21] Q. Huang, C. Zhao, M. Jiang, X. Li, and J. Liang, "Cascade-net: a new deep learning architecture for OFDM detection," [Online]. Available: arXiv:1812.00023v1, preprint, 2018.

[22] Z. Zhao, M. Vuran, F. Guo, and S. Scott, "Deep-waveform: A learned OFDM receiver based on deep complex convolutional networks," arXiv:1810.07181 [eess.SP], 2018.

[23] T. J. O'Shea, K. Karra, and T. C. Clancy, "Learning to communicate: Channel auto-encoders, domain specific regularizers, and attention," in 2016 IEEE International Symposium on Signal Processing and Information Technology (ISSPIT), Dec 2016, pp. 223-228.

[24] T. Q'Shea and J. Hoydis, "An introduction to deep learning for the physical layer," IEEE Tran. on Cognitive Comm. and Networking, vol. 3, no. 4, pp. 563-574, Oct. 2017.

[25] T. Q'Shea, T. Erpek, and T. C. Clancy, "Deep learning-based MIMO communications," arXiv:1707.07980, 2017.

[26] N. Samuel, N. Diskin, and A. Wiesel, "Deep MIMO detection," arXiv preprint arXiv:1706.0115, 2018.

[27] V. Corlay et. al., "Multilevel MIMO detection with deep learning," arXiv:1812.01571, 2018.

[28] H. He et. al., "A model-driven deep learning network for MIMO detection," arXiv:1809.09336, 2018.

[29] C. Huang et. al., "Deep learning for UL/DL channel calibration in generic massive MIMO systems," arXiv:1903.02875, 2019.

[30] T. V. Luong et. al., "Deep learning-based detector for OFDMIM," IEEE Wireless Commun. Lett., vol. 0, no. 0, p. 0, Apr. 2019.

[31] M. Turhan, E. Ozturk, and H. Cirpan, "Deep learning aided generalized frequency division multiplexing," in Proc. 3rd Intl. Balkan Conf. Commun. Networking, Skopje, North Macedonia.

[32] C. Zhang, P. Patras, and H. Haddadi, "Deep learning in mobile and wireless networking: A survey," IEEE Communications Surveys and Tutorials, vol. PP, 032018.

[33] Q. Mao, F. Hu, and Q. Hao, "Deep learning for intelligent wireless networks: Comprehensive survey," IEEE Communications Surveys Tutorials, vol. 20, no. 4, pp. 2595-2621, Fourthquarter 2018.

[34] T. Wang et. al., "Deep learning for wireless physical layer: Opportunities and challenges," arXiv:1710.05312, 2017.

[35] A. Zappone et. al., "Wireless networks design in the era of deep learning: Model-based, AI-based, or both?" arXiv:1902.02647, 2019.

[36] 3GPP, "Base station (BS) radio transmission and reception," tech. spec. 36.104 V14.4.0, Jun. 2017.

[37] K. D. and J. Ba, "Adam: A method for stochastic optimization," arXiv preprint arXiv:1412.6980, 2014.

[38] F. Chollet, M. Vura, F. Guo, and S. Scott, "Keras," https://keras.io, 2015.

[39] M. Abadi and et. al, "Tensorflow: Large-scale machine learning on heterogeneous systems," [Online]. Available: https://www.tensorflow.org/, 2015. 